\section{ORIGINAL RESEARCH}

C. Jung
B.J. Kwon
O.-K. Kwon
S.K. Baik
M.H. Han
J.E. Kim
C.W. Oh

\title{
Intraosseous Cranial Dural Arteriovenous Fistula Treated with Transvenous Embolization
}

\begin{abstract}
BACKGROUND AND PURPOSE: We analyzed the angiographic architecture of intraosseous dural arteriovenous fistulas (DAVFs) and evaluated the use of transvenous embolization for curative treatment.
\end{abstract}

\begin{abstract}
MATERIALS AND METHODS: The study population consisted of 6 patients with intraosseous DAVFs from 3 hospitals. In all of these patients, we retrospectively reviewed the medical records and images, and we were able to confirm the lesions in all patients from CT, MR imaging, and angiographic images. $3 \mathrm{D}$ rotational angiographic coronal source images clearly demonstrated the presence of an intraosseous DAVF in 2 patients.
\end{abstract}

\begin{abstract}
RESULTS: An intraosseous DAVF was located at the upper clivus in 1, the petrous apex in 1, and the lower clivus adjacent to the hypoglossal canal in 4 cases. All of the cases showed the presence of a dilated venous pouch, manifest as an osteolytic lesion on CT and as an intraosseous signal-intensity void on MR images. All patients were treated with transvenous embolization by targeting the dilated venous pouch and its connecting tributaries. Four intraosseous DAVFs were immediately completely embolized. One patient had a residual shunt, but the shunt disappeared 1 month later. One patient presented with a simultaneous DAVF in the ipsilateral cavernous sinus without a significant amount of shunt. None of the patients had procedural complications, and 5 patients recovered from the presenting symptoms.
\end{abstract}

CoNCLUSIONS: An intraosseous DAVF could be completely cured with transvenous embolization. For curative treatment, the intraosseous dilated venous pouch can be the target lesion for endovascular treatment.

A dural arteriovenous fistula (DAVF) can be defined as an abnormal shunt between the meningeal arteries and meningeal veins or sinus or both. ${ }^{1-4}$ Classic DAVFs are situated within the dura adjacent to the venous sinuses, such as the transverse, sigmoid, and cavernous sinuses. ${ }^{5}$ Therefore, classic DAVFs have usually been classified and named according to the location of the involved sinus. Many cases of DAVFs with an extrasinusal location, such as the skull base or the tentorium, have been described in the literature, and a DAVF might occur at any site within the dura. 4,6,7

Intraosseous DAVFs are rare entities. Malik et $\mathrm{al}^{8}$ first described intraosseous DAVFs and found that they were different from classic or extrasinosal DAVFs because the vascular nidus was entirely situated within the bone. They also reported that a DAVF nidus was not found on the dural surface and there were enlarged arteries or veins within the dural surface. The descriptive term "intraosseous DAVF" was used for distinguishing the location of the arteriovenous shunt between

Received October 16, 2008; accepted after revision January 6, 2009.

From the Departments of Radiology (C.J., B.J.K., M.H.H.) and Neurosurgery (0.-K.K., M.H.H., J.E.K., C.W.O.), and Institute of Radiation Medicine (M.H.H.), Seoul National University College of Medicine, Seoul, Korea; Departments of Radiology (C.J.) and Neurosurgery (0.-K.K., C.W.O.), Seoul National University Bundang Hospital, Seongnam, Korea; Departments of Radiology (B.J.K., M.H.H.) and Neurosurgery (J.E.K.), and Clinical Research Institute (M.H.H.), Seoul National University Hospital, Seoul, Korea; and Department of Radiology (S.K.B.), Pusan National University Yangsan Hospital, Busan, Korea.

This study was supported by a grant of the Korea Health 21 R\&D Project, Ministry of Health \& Welfare, Republic of Korea (grant no: A06-0171-B51004-06N1-00040B).

Please address correspondence to Moon Hee Han, MD, PhD, Department of Radiology, Seoul National University Hospital, 101 Daehangno, Jongno-gu, Seoul 110-744, Korea; e-mail: hanmh@snuh.org

Indicates supplemental on-line table.

Indicates open access to non-subscribers at www.ajnr.org

DOI 10.3174/ajnr.A1528

the bone and dura, which was useful for the localization of the lesion for surgical resection. If surgical resection of an intraosseous DAVF was attempted, it would be important to determine the correct location of the nidus. Furthermore, bleeding during surgery could occur due to hypervascularity in the intraosseous compartment of the DAVF.

There have been a few case reports describing intraosseous DAVFs. ${ }^{3,8-12}$ Some patients were treated by surgical resection, and sometimes the DAVFs were treated by endovascular treatment via the transarterial or transvenous route. Transvenous embolization of a DAVF is the most effective option for curative treatment. ${ }^{13}$ Similarly, we thought that an intraosseous DAVF could be completely treated by transvenous embolization, so in this report, we analyzed the angiographic architectures of intraosseous DAVFs and present the outcomes of transvenous endovascular embolization for their treatment.

\section{Materials and Methods}

Six patients (M/F, 4:2; mean age, 58.6 years; age range, $15-82$ years) with intraosseous DAVFs were treated by endovascular techniques in the 3 hospitals and composed the study population. The demographic profiles, symptoms, and signs are summarized in the on-line Table. The patients had different symptoms and signs of a variable duration, but pulsatile tinnitus was the most frequent symptom. Case 1 presented with diplopia and ptosis indicating that the lesion was located at the cavernous sinus. Case 2 had hearing loss at 4 months before admission, which was not a usual symptom for the presence of DAVF.

In all of these patients, we retrospectively reviewed medical records and images for an anatomic analysis of the lesions and the clinical outcomes after endovascular treatment. There were preprocedural CT, MR imaging, and conventional angiographic images available for all these patients. We determined immediate angiographic outcome as residual shunt or complete occlusion after treatment, whether or not residual shunt remained. Follow-up anatomic 

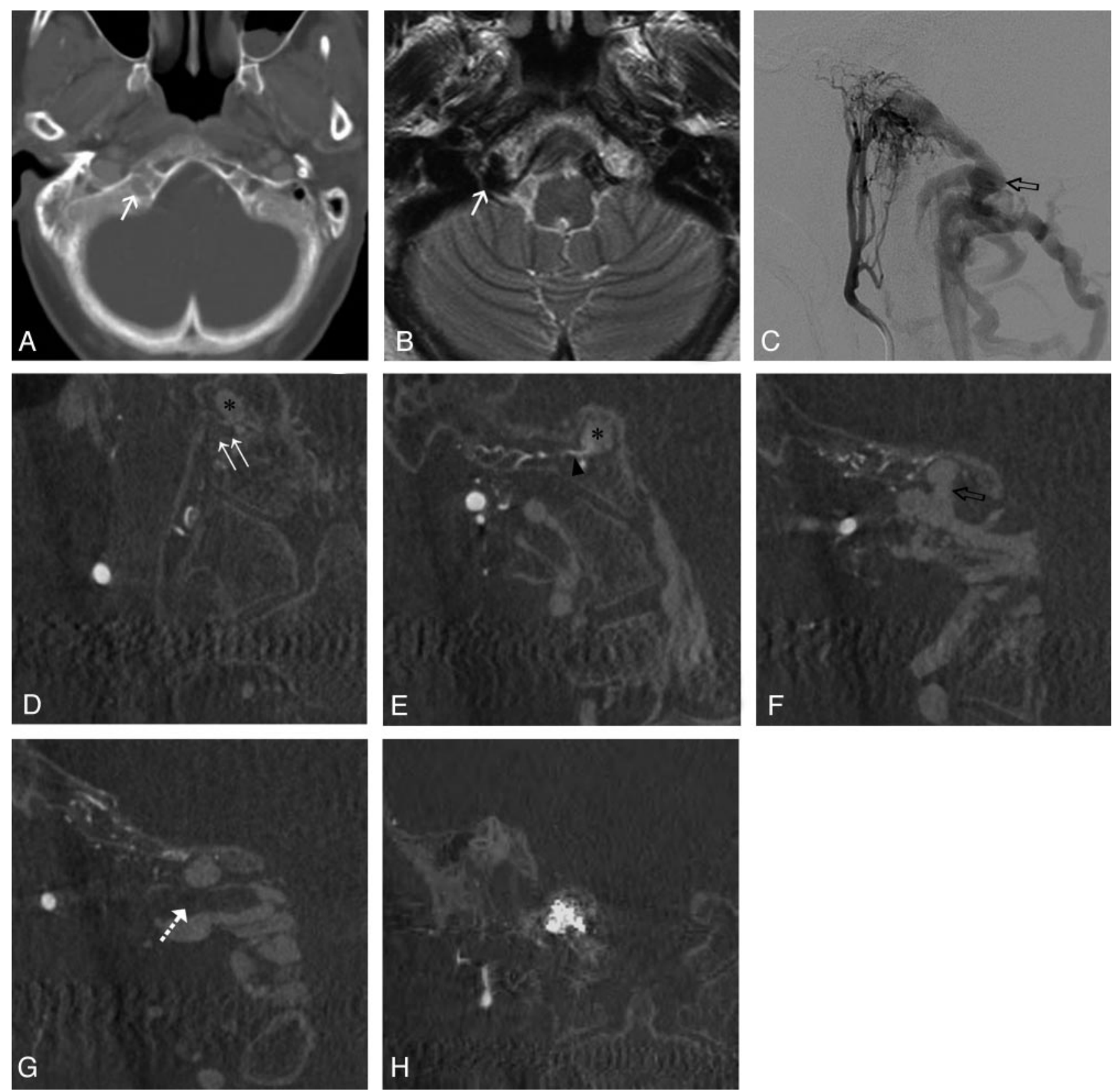

Fig 1. Case 3. Axial noncontrast CT scans with a bone window setting $(A)$ and a T2-weighted axial MR image $(B)$ show the osteolytic lesion and signal-intensity void, respectively (white arrow), which correspond to the intraosseous venous pouch (asterisks in $D$ and $E$ ) and are located at the lower clivus around the hypoglossal canal (double arrow in $D$ ). Selective angiography of the right ascending pharyngeal artery $(C)$ and coronal reconstructed images of rotational angiography $(D-G)$ show a fistula between the artery and dilated venous pouch (arrowhead in $\theta$ ) and venous drainage into vertebral venous system, such as the venous plexus, around the horizontal segment of the vertebral artery (dotted arrow) via the posterior condylar vein lopen arrow in $C$ and $F$. After transvenous embolization, coil was packed within the dilated venous pouch $(H)$.

outcome was evaluated by MR angiography (MRA) or conventional angiography. Follow-up MRA or conventional angiography was available in 5 patients, but there was no follow-up MRA or angiography in 1 patient (case 6).

\section{Results}

\section{Angiographic Details of Intraosseous DAVFs}

We were able to confirm the presence of an intraosseous DAVF in all the patients with the use of CT, MR imaging, and cerebral angiography. The abnormal dilated venous sac within the bone was demonstrated on the CT and MR images, including time-of-flight MRA (TOF-MRA) or coronal source images of $3 \mathrm{D}$ rotational angiography (Figs $1 D-H$ and $2 E$ and $F$ ). In all of the cases, CT or MR imaging showed the dilated venous pouch within the bone. The lesions were manifest as osteolytic lesions within the bony structure as seen on noncontrast CT images with the bone window setting (Figs $1 A$ and $2 A$ ), and the dilated venous pouch as seen with a signal-intensity void on MR images (Fig 1B).

MRA was performed for the all patients except for case 2, and imaging showed the presence of an arteriovenous shunt around the abnormal dilated venous sac. The lesions were located at the upper clivus, petrous apex, or lower clivus around the hypoglossal canal. The most common location of a lesion 

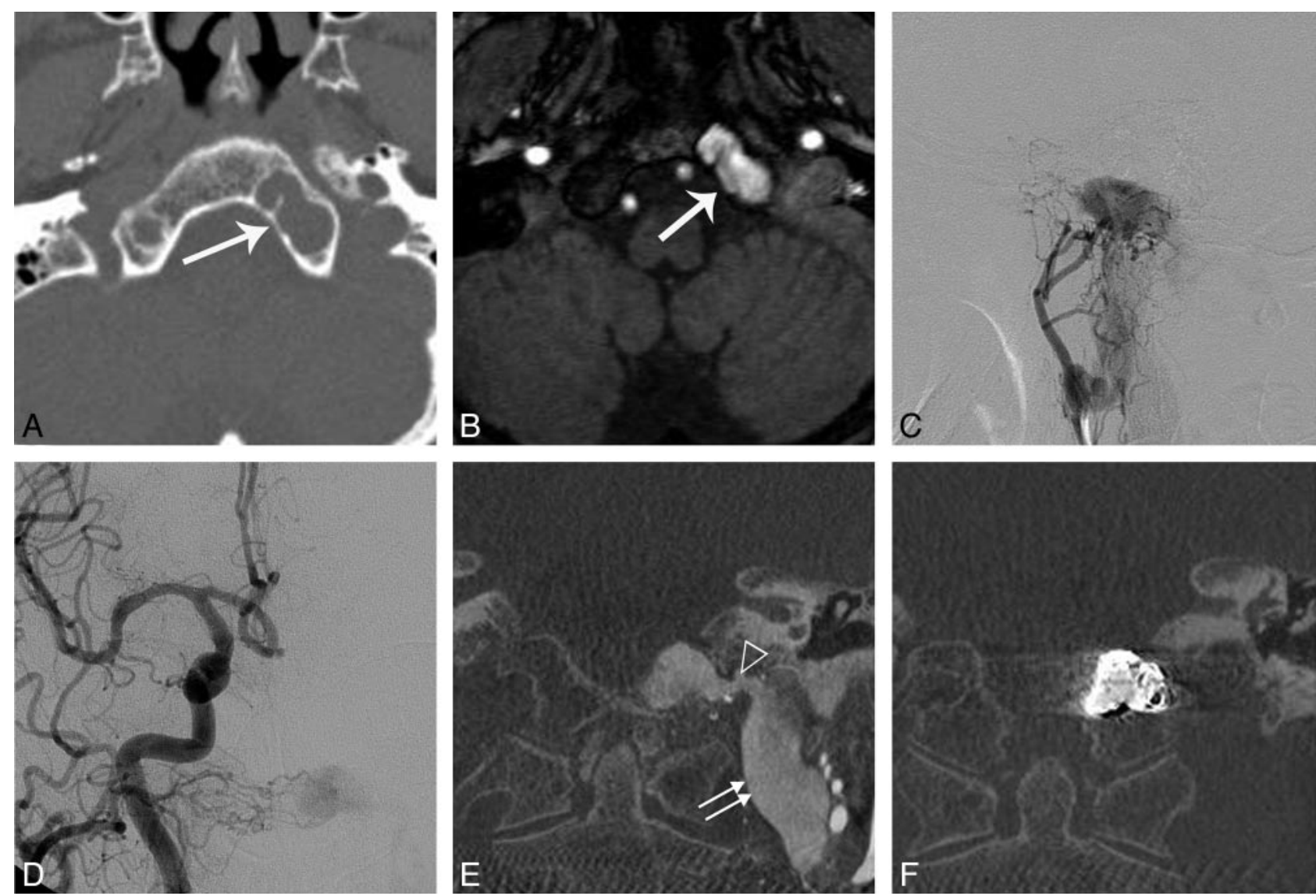

Fig 2. Case 6. The intraosseous venous pouch (arrows in $A$ and $B$ ) was located at the left lower clivus around the hypoglossal canal, and selective angiography of the ascending pharyngeal artery confirmed an arteriovenous shunt $(C)$, which was more correctly localized by the contralateral common carotid angiography $(D)$. The venous connection (open arrowhead) between the intraosseous venous pouch and the internal jugular vein (double arrows) was so obvious on the coronal reconstructed image of rotational angiography ( $E$ ) that the intraosseous venous pouch and venous connection could be completely occluded by the transvenous embolization $(F$.

was the lower skull base around the hypoglossal canal in 4 patients. Conventional angiography showed the arteriovenous shunt in the wall of the abnormally dilated venous pouch. In 2 patients, the osseous lesions were clearly demonstrated on the coronal reconstructed images of $3 \mathrm{D}$ rotational angiography (Integris Allura; Philips Medical Systems, Best, the Netherlands).

As seen on angiography, the intraosseous DAVFs were predominantly fed by the meningeal branches, such as the ascending pharyngeal artery, occipital artery, meningohypophyseal trunk of the internal carotid artery (ICA), and the anterior meningeal artery from the vertebral artery, as expected. The subarcuate artery from the anterior inferior cerebellar artery (AICA) supplied the lesion in case 2. These feeding arteries were focused on the wall of the dilated venous pouch, which was considered the target area of treatment. In cases 3 and 6, coronal source images of $3 \mathrm{D}$ rotational angiography demonstrated that the fistula was located at the wall of the dilated venous pouch (Fig $1 E)$.

The dilated venous pouch was located within the bone and communicated with venous structures around the lesions (Figs $1 F$ and $2 E$ ). In cases 1 and 4 , the DAVFs showed retrograde venous reflux into the cavernous sinus via the inferior petrosal sinus (IPS), which also resulted in leptomeningeal and superior ophthalmic reflux. In cases 3 and 5 in which the lesions were located around the hypoglossal canal, shunt flows drained into the prevertebral and internal epidural venous plexus of the spine via the transosseous emissary vein, the socalled anterior and posterior condylar vein. There was perimedullary reflux via the internal epidural venous plexus in case 5 . Cases 1 and 4 showed cortical venous drainage by the reflux via the IPS and cavernous sinus.

\section{Endovascular Treatment}

All patients were treated via the transvenous approach. It was very important to define and access the abnormal dilated venous pouch with a microcatheter and to know the venous connection with the abnormal dilated venous pouch, such as the IPS, because persistent symptoms could result if the arteriovenous shunt was not completely treated and if the symptomatic venous connection was completely occluded. It was especially difficult to access the lesion in case 1 due to the tortuous and small trajectory of the vein; a microcatheter compatible with 0.010 sized device was used and the lesion was occluded with detachable coils. It was not as difficult to approach the venous sac in the other patients; the fistulas of the 5 patients were occluded with the use of pushable fibered coils, except case 1 .

Immediately after treatment, the shunt was completely occluded in 4 patients (cases $2,4-6$ ). In case 3 , the residual shunt was spontaneously occluded as seen on 1-month follow-up angiography. In case 1, there were 2 DAVFs, 1 of which was 
located at the upper clivus as an intraosseous DAVF and the other, at the posterior portion of ipsilateral cavernous sinus as a classic DAVF. Therefore, we could completely treat the upper clival lesion, but a residual weak shunt of DAVF in the ipsilateral cavernous sinus remained. This lesion became obvious after treatment, and we thought that the DAVF of the cavernous sinus was a simultaneous lesion that was not related to the symptoms. This lesion not only remained unchanged as seen on a 24-month MRA but also was not symptomatic. Patients were clinically followed up for a mean of 10.2 months (range, 1-24 months). All patients improved clinically, but the symptom of hearing loss in case 2 persisted at the 6-month follow-up. The hearing loss was considered irreversible, probably due to the long symptom duration of 4 months. There were no other procedural complications or additional symptoms and signs after treatment in all patients.

\section{Discussion}

DAVF can be defined as an abnormal shunt between the meningeal artery and vein or sinus or both within the dura. ${ }^{1-4}$ The term "intraosseous DAVF" may be a misnomer and "intraosseous AVF" might be more accurate. ${ }^{3}$ Malik et al $^{8}$ first described 2 cases of DAVFs that were treated by surgical excision. The lesions were completely removed by resection of the pathologic bony portion. The lesion of the "intraosseous DAVF" had the nidus exclusively located within the bone, with a venous drainage pattern similar to that of a classic DAVF as seen on angiography.

Geibprasert et al $^{14}$ recently defined DAVFs as a group of diseases that share involvement of the epidural space, adjacent dura mater, and bony structures. They adopted this definition for the classification of a spinal DAVF and an intracranial DAVF, according to the evolution and embryologic development of the venous drainage of the central nervous system and surrounding structures, such as vertebrae, skull base, and calvaria. According to this study, the dura and surrounding bone were closely related, and the DAVF could involve 1 of 2 compartments or both, though its etiology was uncertain.

Anatomically, the meningeal artery also supplies the adjacent bony structure, and the dura can share the venous drainage with the adjacent bony structure. ${ }^{15}$ In intraosseous DAVFs, the fistula is seen between the meningeal arteries and intraosseous diploic veins or the transosseous emissary veins, such as the anterior condylar vein; and intraosseous DAVFs have a clinical manifestation and natural course of venous drainage pattern similar to that of the classic DAVFs. ${ }^{6,11}$ It would be more suitable to define an intracranial DAVF as an abnormal arteriovenous connection between the meningeal artery and vein or sinus or both within the dura or skull or both, for the inclusion of an intraosseous DAVF. Therefore, we consider that "intraosseous DAVF" is the proper and descriptive term and is a subtype of an intracranial DAVF in which the fistula is predominantly located at the intraosseous compartment.

There have been several reports of intraosseous DAVFs described as hypoglossal canal DAVFs and anterior or posterior condylar vein DAVFs. ${ }^{9,10,12}$ These lesions could possibly be intraosseous DAVFs, but not always. Ernst et $\mathrm{al}^{9}$ described 3 cases of DAVFs that involved the anterior condylar vein within the hypoglossal canal. These cases seemed to be similar to in- traosseous DAVFs because the lesions showed the presence of an intraosseous venous pouch adjacent to the hypoglossal canal and nidus within the bone on MR imaging and MRA source images. Tanoue et $\mathrm{al}^{11}$ also described 2 similar cases in which the anterior condylar vein was involved. The first lesion involved the intracranial portion of the anterior condylar vein with reflux into the perimedullary vein and showed congestive myelopathy in the upper cervical spinal cord. It had no intraosseous component of a fistula. However, the second case involved the anterior condylar vein within the hypoglossal canal with an intraosseous venous pouch, and the lesion was treated completely with endovascular embolization via the transvenous approach. Recently, Kiyosue et $\mathrm{al}^{10}$ reported 1 case of a DAVF located at the posterior condylar canal. They also showed the presence of an intraosseous venous pouch adjacent to the posterior condylar canal, which was confirmed on posttreatment CT images. The 2 veins were transosseous emissary veins in the posterior cranial fossa. Therefore, we thought that these veins could be a route for venous drainage of any DAVFs involving the adjacent dural wall or an intraosseous DAVF.

In this report, we have described 2 fistulas that were located within the upper clivus and petrous apex. In these areas, though there were no specific nominated venous structures, it is known that transosseous emissary veins are abundant and complex in the skull base, ${ }^{16,17}$ and it could be inferred that an intraosseous DAVF could occur in these areas related to the transosseous emissary vein.

A dilated venous pouch within the bone of an intraosseous DAVF was manifest as an osteolytic lesion on CT images, and signal-intensity enhancement within the bone was seen on TOF-MRA source images. ${ }^{3}$ For a classic DAVF, the venous structure could be stenotic or dilated due to an arteriovenous shunt. ${ }^{18}$ A dilated venous pouch could also be a secondary change from a fistula; however, because the shunt and related venous structure in the intraosseous DAVF are located within the bone, a dilated venous pouch within the bone could be an indirect sign on the CT or MR images.

Chen et $\mathrm{al}^{3}$ described the MR imaging findings of 2 patients with intraosseous DAVFs. For both patients, the nidus was located at the clivus. MR imaging and MRA source images showed the presence of an intraosseous venous pouch within the clivus. Additionally, the diploic space was well-enhanced on a postcontrast T1-weighted image, which is suggestive of intradiploic venous hypertension caused by an intraosseous DAVF. CT can help demonstrate the presence of the osteolytic portion secondary to the dilated venous pouch before treatment. The cases in the present study were similar to previously reported cases in which the fistula was located within the bone and an intraosseous venous pouch was also noted on CT angiography or MRA source images. We could demonstrate the intraosseous lesions in 2 patients on coronal source images of $3 \mathrm{D}$ rotational angiography, which are considered to provide the most accurate diagnostic information for the identification of intraosseous DAVFs by showing enhanced fine vascular structures and bone changes together (Fig 1E).

Intraosseous DAVFs in the posterior fossa can be treated by surgical resection, endovascular occlusion via the transarterial or transvenous route, or radiation or a combined technique. Surgical resection for a posterior fossa DAVF achieved cura- 
tive results in several reports, but surgery was usually selected as a secondary choice after endovascular treatment because of the risk. ${ }^{2,4,8}$

In selected cases, transarterial embolization of a DAVF in other locations could be curative, ${ }^{12}$ but in the case of an intraosseous DAVF, most of the fistulas recurred after transarterial embolization. ${ }^{3,8}$ The ascending pharyngeal artery (APA) was the main feeder for the intraosseous DAVFs in the previous reports and in the present cases. The APA not only supplied the DAVF but also supplied the lower cranial nerves such as the glossopharyngeal, vagus, and hypoglossal nerves. ${ }^{15}$ Transarterial embolization of the APA could produce a risk of lower cranial nerve palsy. ${ }^{9}$ Therefore, transarterial embolization of the DAVF might be a palliative option for symptomatic relief when a curative transvenous embolization is not possible technically.

Transvenous embolization of intraosseous DAVFs could be curative as ascertained in several studies and in the present cases. $^{9-12}$ We thought that it was very important to define the fistula focus as manifest by the dilated venous pouch within the bone. In cases with a lesion involving the hypoglossal canal, contralateral external carotid artery, or common carotid artery angiography could be helpful for localization of the lesion. ${ }^{9,10}$ Source images of 3D rotational angiography could provide more accurate information than other imaging techniques. We were able to achieve complete occlusion of the DAVFs in all of the patients without any complications after embolization of the dilated venous pouch. Although the use of transvenous embolization would be safe, it could also cause complications. There was 1 case reported of a hypoglossal canal DAVF complicated by permanent hypoglossal nerve palsy after transvenous embolization in the dilated venous pouch. ${ }^{12}$ Because the hypoglossal canal is a tubular canal with limited space, a packed coil mass within the canal could produce a mass effect on the hypoglossal nerve and cause nerve palsy.

\section{Conclusions}

The strategy and method of the treatment of intraosseous DAVFs is not different from those in classic DAVFs. Despite our limited experience, we can conclude that transvenous embolization, accurately targeted for the dilated venous pouch in an intraosseous DAVF, can be a safe and curative method, and a good clinical outcome can be expected immediately or dur- ing the follow-up period. For curative treatment, it is very important to analyze the angiographic architecture correctly. It would be helpful to determine if the intraosseous dilated venous pouch could be a target lesion; then it can be correctly localized by the use of coronal source images of $3 \mathrm{D}$ rotational angiography.

\section{References}

1. Sarma D, ter Brugge K. Management of intracranial dural arteriovenous shunts in adults. Eur J Radiol 2003;46:206-20

2. McDougall CG, Halbach VV, Dowd CF, et al. Dural arteriovenous fistulas of the marginal sinus. AJNR Am J Neuroradiol 1997;18:1565-72

3. Chen CJ, Wai YY, Wang LJ, et al. MRI of intraosseous dural arteriovenous malformation: findings in two cases. J Comput Assist Tomogr 2001;25:133-36

4. Mahmood A, Malik GM. Dural arteriovenous malformations of the skull base. Neurol Res 2003;25:860-64

5. Chaudhary MY, Sachdev VP, Cho SH, et al. Dural arteriovenous malformation of the major venous sinuses: an acquired lesion. AJNR Am J Neuroradiol 1982;3:13-19

6. Piske RL, Lasjaunias P. Extrasinusal dural arteriovenous malformations: report of three cases. Neuroradiology 1988;30:426-32

7. Ito J, Imamura H, Kobayashi K, et al. Dural arteriovenous malformations of the base of the anterior cranial fossa. Neuroradiology 1983;24:149-54

8. Malik GM, Mahmood A, Mehta BA. Dural arteriovenous malformation of the skull base with intraosseous vascular nidus: report of two cases. J Neurosurg 1994;81:620-23

9. Ernst R, Bulas R, Tomsick T, et al. Three cases of dural arteriovenous fistula of the anterior condylar vein within the hypoglossal canal. AJNR Am J Neuroradiol 1999;20:2016-20

10. Kiyosue $\mathrm{H}$, Okahara $\mathrm{M}$, Sagara $\mathrm{Y}$, et al. Dural arteriovenous fistula involving the posterior condylar canal. AJNR Am J Neuroradiol 2007;28:1599-601

11. Tanoue S, Goto K, Oota S. Endovascular treatment for dural arteriovenous fistula of the anterior condylar vein with unusual venous drainage: report of two cases. AJNR Am J Neuroradiol 2005;26:1955-59

12. Manabe S, Satoh K, Matsubara S, et al. Characteristics, diagnosis and treatment of hypoglossal canal dural arteriovenous fistula: report of nine cases. Neuroradiology 2008;50:715-21

13. Halbach VV, Higashida RT, Hieshima GB, et al. Transvenous embolization of dural fistulas involving the cavernous sinus. AJNR Am J Neuroradiol 1989;10:377-83

14. Geibprasert S, Pereira V, Krings T, et al. Dural arteriovenous shunts: a new classification of craniospinal epidural venous anatomical bases and clinical correlations. Stroke 2008;39:2783-94. Epub 2008 Jul 17

15. Martins C, Yasuda A, Campero A, et al. Microsurgical anatomy of the dural arteries. Neurosurgery 2005;56:211-51

16. Okudera T, Huang YP, Ohta T, et al. Development of posterior fossa dural sinuses, emissary veins, and jugular bulb: morphological and radiologic study. AJNR Am J Neuroradiol 1994;15:1871-83

17. San Millan Ruiz D, Gailloud P, Rufenacht DA, et al. The craniocervical venous system in relation to cerebral venous drainage. AJNR Am J Neuroradiol 2002;23:1500-08

18. Kwon BJ, Han MH, Kang HS, et al. MR imaging findings of intracranial dural arteriovenous fistulas: relations with venous drainage patterns. AJNR Am J Neuroradiol 2005;26:2500-07 